CARDIOVASCULAR MEDICINE

\title{
Allopurinol reduces B-type natriuretic peptide concentrations and haemoglobin but does not alter exercise capacity in chronic heart failure
}

\author{
A D Gavin, A D Struthers
}

Heart 2005;91:749-753. doi: 10.1136/hrt.2004.040477

See end of article for authors' affiliations .....................

Correspondence to: Professor Allan D Struthers, Division of Medicine \& Therapeutics, Ninewells Hospital, Dundee DDI 9SY, UK; a.d.struthers@ dundee.ac.uk

Accepted 17 August 2004
Objective: To study whether the effect of allopurinol on improvement of endothelial dysfunction in chronic heart failure (CHF) translates into improved exercise capacity and to examine whether allopurinol also improves B-type natriuretic peptide (BNP), the other important prognostic marker of CHF.

Design: Randomised, double blind, placebo controlled crossover trial.

Setting: Teaching hospital.

Patients: 50 patients with CHF (New York Heart Association functional classes II and III) were recruited. Interventions: 50 patients with CHF were randomly assigned to three months' treatment with allopurinol (300 mg/day) or placebo. At two and three months into treatment, they underwent a modified Bruce exercise protocol and a six minute walk test. Blood was taken for BNP and haemoglobin analysis.

Results: Neither exercise test was altered by allopurinol. However, plasma BNP concentrations fell significantly $(p=0.035$ ) with allopurinol (1 $1.9 \mathrm{pmol} / \mathrm{l})$ versus placebo (14.4 pmol/l). Haemoglobin concentrations also fell highly significantly with allopurinol $(p=0.001)$.

Conclusions: An important negative finding is that despite high hopes for it, allopurinol had no effect on exercise capacity in CHF. On the other hand, allopurinol did reduce BNP, which is the best available surrogate marker for prognosis in $\mathrm{CHF}$.
$\mathrm{T}$ here is now a compelling body of evidence that allopurinol improves endothelial dysfunction in a wide variety of patients with oxidative stress. Xanthine oxidase inhibition improved endothelial dysfunction in patients with hypercholesterolaemia or diabetes, in cigarette smokers, and in three separate studies of heart failure. ${ }^{1-5}$ Overall seven studies have shown that allopurinol improves endothelial dysfunction whereas only two have disagreed. ${ }^{16}$ Importantly, all three studies of heart failure were positive. ${ }^{45}$

The key question that now arises is: what are the consequences of allopurinol improving endothelial dysfunction in chronic heart failure (CHF)? One distinct possibility highlighted in a review article in Circulation is that endothelial dysfunction may actually be causing some of the exercise intolerance of $\mathrm{CHF}^{7}$ Drexler $^{7}$ further speculated that treatments that improve endothelial dysfunction in CHF should also improve exercise capacity and he quoted L-arginine, vitamin $\mathrm{C}$, and physical training as examples that supported this hypothesis. ${ }^{8}$ We therefore investigated whether this was the case for allopurinol.

The other main consequence may be that allopurinol's ability to improve endothelial dysfunction may also translate into an improved prognosis. ${ }^{9}$ The assertion that there is a link between endothelial dysfunction and future cardiovascular events is based on two large bodies of evidence. ${ }^{10}$ Firstly, seven studies have now shown that spontaneous endothelial dysfunction predicts future cardiovascular events. ${ }^{11-17}$ Secondly, treatment induced improvements in endothelial dysfunction usually parallel treatment induced improvements in cardiovascular events and mortality. This is true in the cases of aspirin, statins, angiotensin converting enzyme (ACE) inhibitors, spironolactone, and angiotensin antagonists (LIFE, losartan for endpoint reduction in hypertension study ${ }^{18}$ ). However, this is not always the case-for example, with hormone replacement therapy. Therefore, it would be of interest to see whether allopurinol also improves other prognostic markers to gain some insight into whether allopurinol's ability to improve endothelial dysfunction is likely to follow promising examples such as ACE inhibitors or to follow inauspicious examples such as hormone replacement therapy. In repeated studies, the strongest independent prognostic marker in heart failure is nearly always B-type natriuretic peptide (BNP). ${ }^{19}$ Hence, if allopurinol improves both BNP and endothelial dysfunction, this would add impetus to the idea that allopurinol may improve prognosis in CHF.

\section{METHODS \\ Patients}

Fifty patients with CHF (New York Heart Association functional classes II and III) and documented left ventricular (LV) systolic dysfunction on echocardiogram or radionuclide ventriculogram were recruited from the heart failure clinic and from a database of patients with echocardiographically confirmed LV dysfunction. The study protocol was approved by the Tayside committee on medical research ethics and each patient provided written informed consent. This study complied with the Declaration of Helsinki. For our protocol, we basically followed all the advice on how to do exercise studies in CHF as suggested by the authoritative review article of Narang and colleagues. ${ }^{20}$ This includes their recommendations on end points, sample size, and crossover studies.

The recruits underwent screening by clinical history and physical examination, and routine haematological and biochemical analyses. Before entering the study all patients performed a modified Bruce exercise tolerance test and six minute walk test to familiarise themselves with the protocols.

Abbreviations: $\mathrm{ACE}$, angiotensin converting enzyme; $\mathrm{BNP}$, B-type natriuretic peptide; CHF, chronic heart failure; COPERNICUS, carvedilol prospective randomised cumulative survival; $\mathrm{CV}$, coefficient of variation; $\mathrm{LV}$, left ventricular 
Patients were excluded if they were unable to perform the exercise tests, if they had a history of gout and were taking regular allopurinol, if they had had a previous adverse reaction to allopurinol, or if they had uncontrolled hypertension. All recruits had been clinically stable for at least six weeks before enrolment in the study with no modification to drug treatment. We chose not to measure oxygen consumption because it can be uncomfortable for patients and because Narang and colleagues ${ }^{20}$ concluded that there is no evidence of its superiority to exercise duration alone. Indeed, they felt that the cumbersome nature of its measurement was possibly interfering with the results of exercise testing and increasing the tendency to negative results.

\section{Study protocol}

After initial screening, blood was taken for analysis of BNP, cholesterol, haemoglobin, and highly sensitive $\mathrm{C}$ reactive protein. Patients then underwent a baseline modified Bruce exercise tolerance test and six minute walk test. Recruits were then randomly assigned in a crossover fashion to double blind treatment for three months with either $300 \mathrm{mg}$ oral allopurinol or matching placebo daily with a one month washout in between. During each three month treatment period, patients underwent an exercise tolerance test and six minute walk test, and serum uric acid, plasma BNP, cholesterol, and highly sensitive $\mathrm{C}$ reactive protein as well as biochemical and haematological parameters were measured after two months and three months of treatment.

The BNP samples were stored at $-70^{\circ} \mathrm{C}$. BNP was extracted from plasma on $\mathrm{C} 18$ columns and then measured by radioimmunoassay (Bachem, St Helens Merseyside, UK; interassay coefficient of variation (CV) $15.5 \%$, intra-assay CV $12.2 \%$ ). We have been measuring BNP in this way for 12 years.

The highly sensitive $C$ reactive protein samples were left for 20 minutes at room temperature to allow clot retraction before spinning as above and were then stored at $-20^{\circ} \mathrm{C}$. Highly sensitive $\mathrm{C}$ reactive protein was measured by enzyme immunoassay with a kit from Kalon Biological Ltd (Aldershot, UK; interassay CV 8.4\%, intra-assay CV 4.5\%).

The modified Bruce exercise tolerance test was performed according to the American Heart Association's guidelines for clinical exercise testing with an electrically driven treadmill (Marquette 200, Marquette Medical Systems, Milwaukee, Wisconsin, USA) and ECG recording system with built-in automatic arrhythmia sensing (Marquette Case 16, Marquette Medical Systems). ECG was monitored continuously throughout the procedure and the blood pressure was checked manually at baseline and at three minute intervals with a regularly calibrated Accuson mercury manometer (AC Cosser \& Son (Surgical) Ltd, UK) placed at the level of the patient's heart. Standardised instructions were given on how to perform the test and a brief demonstration was given. Patients were instructed to exercise until fatigue or dyspnoea prevented them from continuing or until instructed to stop. Exercise duration, symptoms, and reason for discontinuing the test were recorded, as were peak heart rate and blood pressure response.

The six minute walk test was performed after a 30 minute rest period in a long, flat, straight corridor with a $20 \mathrm{~m}$ length marked out every $2 \mathrm{~m}$ and cones at the turnaround points. Patients were instructed to walk as far as possible for six minutes around the cones and were informed when they had walked for four minutes and when the test had been completed. No other words of encouragement were given. The distance covered and number of rest periods taken were recorded.

All data were analysed by analysis of variance including an analysis to see whether there was any treatment order effect.

\begin{tabular}{|c|c|}
\hline \multicolumn{2}{|l|}{ Characteristic } \\
\hline Age (years) & $67(9)$ \\
\hline Men/women & $34 / 9$ \\
\hline NYHA class (II/IIII) & $31 / 12$ \\
\hline \multicolumn{2}{|l|}{ Cause of CHF } \\
\hline Ischaemic & 31 \\
\hline Cardiomyopathy & 4 \\
\hline Valvar heart disease & 2 \\
\hline Other & 6 \\
\hline \multicolumn{2}{|l|}{ Drug treatment } \\
\hline ACE inhibitor & $38(89 \%)$ \\
\hline Ang II antagonist & $3(7 \%)$ \\
\hline$\beta$ Blocker & $23(54 \%)$ \\
\hline Spironolactone & $13(30 \%)$ \\
\hline $\begin{array}{l}\text { Nitrate } \\
\text { lat }\end{array}$ & $12(28 \%)$ \\
\hline Calcium antagonist & $6(14 \%)$ \\
\hline Digoxin & $13(30 \%)$ \\
\hline Statin & $26(60 \%)$ \\
\hline Aspirin & $29(67 \%)$ \\
\hline Clopidogrel & $4(9 \%)$ \\
\hline History of diabetes mellitus & $5(11.6 \%)$ \\
\hline History of hypertension & 15 (34.9\%) \\
\hline History of atrial fibrillation & $12(28 \%)$ \\
\hline \multicolumn{2}{|l|}{ Smoking history } \\
\hline Never & 14 \\
\hline Former & 19 \\
\hline Current & 9 \\
\hline \multicolumn{2}{|c|}{$\begin{array}{l}\text { Data are mean (SD) or number (\%). } \\
\text { ACE, angiotensin converting enzyme; Ang, angiotensin; } \\
\text { CHF, chronic heart failure; NYHA, New York Heart } \\
\text { Association. }\end{array}$} \\
\hline
\end{tabular}

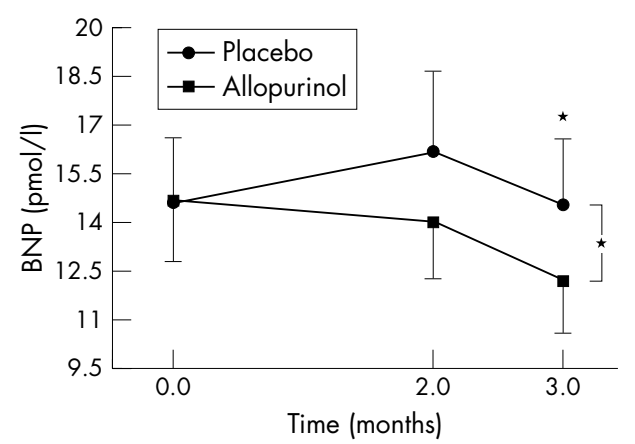

Figure 1 Effect of allopurinol on plasma B-type natriuretic peptide (BNP) concentrations in patients with chronic heart failure (CHF). Data are mean (SEM). * $p<0.05$.

If no treatment order was seen but analysis of variance was significant, two and three month data were analysed by paired $t$ tests to see which individual time points were significant.

\section{RESULTS}

Of the original recruited patients seven did not complete the study. Two patients developed a skin rash while receiving allopurinol. While receiving placebo, one patient developed alopecia, one developed severe back pain, one developed diabetes, one withdrew for personal reasons, and one died. Table 1 shows the baseline characteristics of patients who completed the study. No treatment order effect was seen for any of the parameters measured in this study.

\section{Effect of allopurinol on BNP}

Overall there was a significant (by analysis of variance) reduction in BNP with allopurinol $(\mathrm{p}=0.035)$ (fig $\mathrm{l})$. No significant correlation was seen between the reduction in 


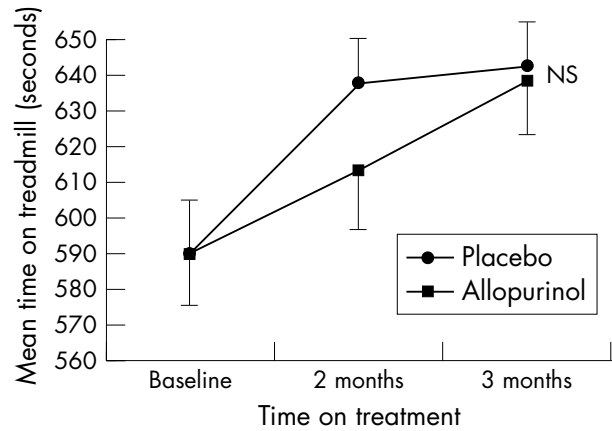

Figure 2 Effect of allopurinol on treadmill performance of patients with CHF. Data are mean (SEM). NS, not significant.

BNP concentration and the reduction in serum uric acid concentration $(\mathrm{p}=0.582)$.

\section{Effect of allopurinol on exercise performance}

There was no significant difference in exercise performance as assessed by either the exercise tolerance test $(p=0.297)$ (fig 2) or the six minute walk test $(p=0.170)$ (fig 3 ) between the active and placebo arms of the study.

\section{Effect of allopurinol on cholesterol}

There was a small but significant reduction (analysis of variance) in total cholesterol concentration with allopurinol and the two and three month allopurinol data were both individually significant (fig 4). No significant change in high density lipoprotein cholesterol $(\mathrm{p}=0.910)$ or in the ratio of total to high density lipoprotein cholesterol was seen.

\section{Effect of allopurinol on other parameters}

As would be expected there was a significant reduction in plasma uric acid concentration with allopurinol treatment (268 (123) $\mu \mathrm{mol} / \mathrm{l})$ compared with placebo (445 (115) $\mu \mathrm{mol} / \mathrm{l}$, $p=0.0001)$. There was no significant effect on potassium $(p=0.869)$, urea $(p=0.796)$ or creatinine $(p=0.975)$, suggesting no direct effect from allopurinol on renal function. However, a two way between group analysis to examine the effect of allopurinol on haemoglobin found a highly significant reduction in haemoglobin in the group receiving allopurinol $(\mathrm{p}=0.001)$ with no significant effect on neutrophil count $(\mathrm{p}=0.419)$ or platelet count $(\mathrm{p}=0.206)$ (fig 5$)$. Highly sensitive $\mathrm{C}$ reactive protein did not change with allopurinol, nor did blood pressure, nor the results of the Minnesota living with heart failure questionnaire (data not shown).

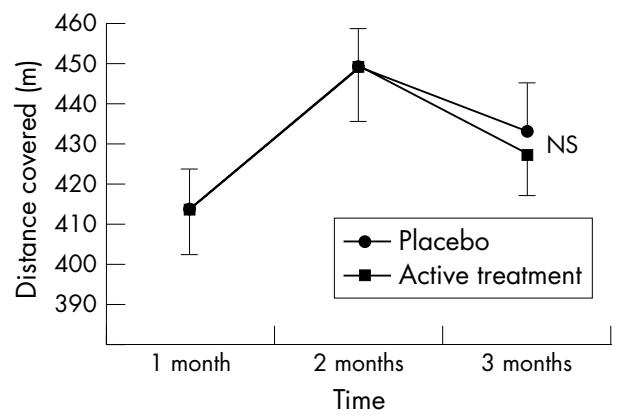

Figure 3 Effect of allopurinol on the six minute walk test in patients with CHF. Data are mean (SEM).

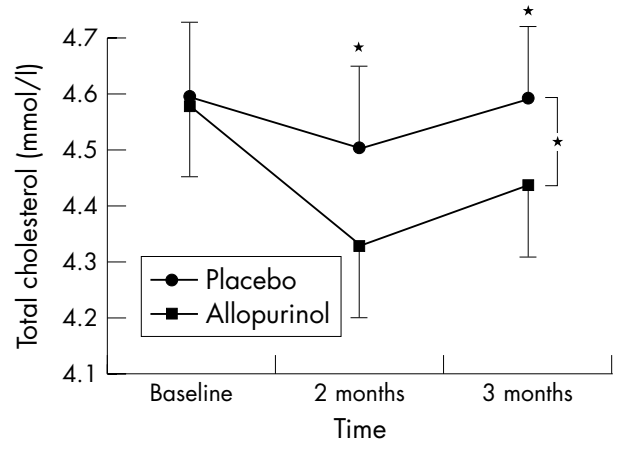

Figure 4 Effect of allopurinol on total cholesterol in patients with CHF. Data are mean (SEM). * $\mathrm{p}<0.05$.

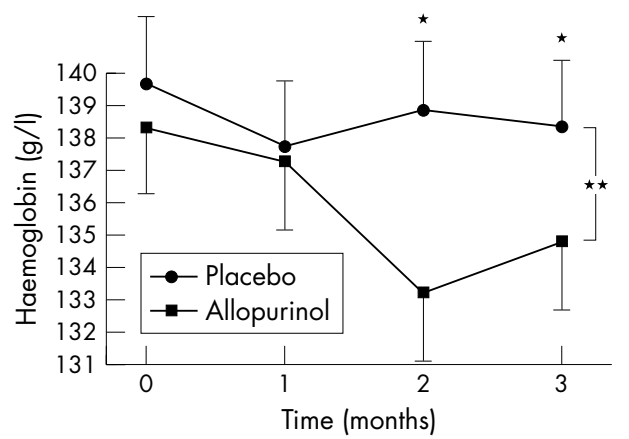

Figure 5 Effect of allopurinol on haemoglobin $(\mathrm{Hb})$ in patients with CHF. Data are mean (SEM). ${ }^{*} \mathrm{p}<0.05 ;{ }^{* *} \mathrm{p}<0.001$.

\section{DISCUSSION}

Before this study, there had been high hopes that allopurinol would improve exercise capacity in CHF and therefore our negative finding is an important negative. ${ }^{21}$ This expectation had been based not only on allopurinol's ability to improve endothelial function in the microcirculation supplying oxygen to exercising skeletal muscles, but also on allopurinol's ability to improve the efficiency of LV contraction. ${ }^{22}$ However, in this study, we used the exercise end points advocated by Narang and colleagues ${ }^{20}$ and measured both maximum and submaximal exercise capacity. We found no trend whatsoever towards improvement at either of the two time points in either exercise end point, despite this study being powered to detect a $23 \%$ improvement in exercise capacity. Our negative results on exercise capacity do, however, agree with our negative results on the Minnesota quality of life questionnaire. This may illustrate how little we understand the determinants of exercise capacity or quality of life of patients with heart failure. Alternately, perhaps in this case, some other effect may have detracted from the effect of the improved central and peripheral haemodynamic function on exercise capacity. One possibility is that the allopurinol induced slight reduction in haemoglobin (and hence oxygen supply to exercising muscles) offset the other benefits, as far as exercise was concerned. Another possibility is that these patients were all receiving optimal treatment for CHF, making their exercise capacity so good that it was difficult to effect a measurable improvement.

There is a huge amount of data that plasma BNP is an excellent prognostic marker not only in patients with heart failure but also in patients with acute coronary syndromes and even in normal populations. ${ }^{19}{ }^{23-25}$ Indeed, in multivariate analysis, BNP almost always outperforms any other 
prognostic marker. ${ }^{19}$ Furthermore, in heart failure, treatments that improve prognosis also consistently reduce BNP by around the same amount as seen here-for example, ACE inhibitors, spironolactone, and chronic $\beta$ blockade. For that reason, our observation that allopurinol reduces BNP is a promising prognostic sign, especially given that allopurinol improves another major indicator of prognosis-that is, endothelial dysfunction. Intriguingly, in terms of surrogates for prognosis, BNP and endothelial function are complementary, as one generally reflects disease progression (BNP) and the other reflects the risk of sudden thrombotic events, and these probably are the two main modes of death in CHF.

It is worth commenting on the concentrations of BNP seen here. With this same assay, we showed previously that the best diagnostic cut off BNP concentration for heart failure was $22.2 \mathrm{pmol} / \mathrm{l}(72 \mathrm{pg} / \mathrm{ml}) .{ }^{26}$ It is well established that BNP concentrations can often be restored to below this cut off and into the "normal" range when optimal treatment is administered, which is probably because most heart failure medications favourably remodel the left ventricle (ACE inhibitors, $\beta$ blockers, spironolactone). ${ }^{27}$ In any case, the BNP concentrations seen here $(\sim 14 \mathrm{pmol} / \mathrm{l})$ are still well above our mean concentration in normal controls ( $4.5 \mathrm{pmol} /$ 1). Hence, our BNP concentrations are consistent with what we usually find for optimally treated patients with CHF in the 21 st century. Furthermore, recent Framingham data suggest that reductions in BNP within the even lower and truly "normal" range may well have prognostic benefit. ${ }^{28}$

It is intriguing to speculate why allopurinol reduces BNP. Reduced BNP implies reduced intracardiac pressure and volume. There are many possible contributors to this. Firstly, improved peripheral vessel endothelial function should reduce LV afterload and hence improve LV pressure in the short term and reduce adverse LV remodelling in the longer term. Secondly, improved coronary endothelial function should improve myocardial blood flow and reduce ischaemia, and so improve LV function. Thirdly, allopurinol induced improvements in LV contraction efficiency may lower BNP. ${ }^{22}$ Fourthly, superoxide anions per se can mediate adverse LV remodelling and allopurinol should reduce this effect, since superoxide anions are a major product of xanthine oxidase activity. ${ }^{29}$ Indeed, many other antioxidants have already been shown to improve LV function. ${ }^{30-38}$ In fact, recently in an animal model, allopurinol itself was shown to greatly improve LV function and remodelling. ${ }^{39}$ Allopurinol induces improvements in endothelial dysfunction and in LV contraction efficiency very quickly, even after single doses, which may explain why intracardiac pressure and BNP were back to their original values after only one month of withdrawal of allopurinol. $^{322}$ Therefore, given all the experimental work described above, it is not that surprising that we found allopurinol to reduce BNP. Indeed, most other treatments that improve endothelial function also improve LV function and remodelling, and so reduce BNP (for example, ACE inhibitors, spironolactone, angiotensin blockers, statins, and exercise training) and hence it is not that surprising that allopurinol does the same. ${ }^{33} 40$

Our finding that allopurinol reduced BNP is bolstered by our finding that allopurinol reduced haemoglobin significantly. It is important to note that other drugs that improve prognosis in CHF also reduce haemoglobin. This applies to both ACE inhibitors and $\beta$ blockers as shown in the COPERNICUS (carvedilol prospective randomised cumulative survival) trial. ${ }^{41}$ In both cases this is probably because they improve LV function and remodelling, which then decrease intracardiac pressure and hence BNP. The fall in BNP then causes haemoglobin concentration to fall because one of the main purposes of natriuretic peptides is to produce haemoconcentration. This was seen in the original landmark paper of de Bold..$^{42}$ BNP induced contraction of the plasma volume is a compensatory mechanism to decrease preload when it is increased, but such a compensatory mechanism may no longer be necessary if LV function is improved by some other treatment. In this way, drugs that improve LV function and remodelling should decrease both BNP and haemoglobin concentrations as has already been seen for ACE inhibitors and $\beta$ blockers. Therefore, allopurinol is following in the footsteps of the two main agents that improve prognosis in CHF. Allopurinol induced falls in BNP and haemoglobin may therefore signify that allopurinol reduces intracardiac pressure and volume, and so also improves LV function and remodelling. Clearly this hypothesis now needs to be investigated specifically.

It is worth emphasising that drug induced intraindividual changes in haemoglobin are very different from interindividual differences in haemoglobin in CHF populations. In CHF populations, the haemoglobin and mortality curve is $\mathrm{U}$ shaped. Frankly low haemoglobin concentrations probably relate to cytokine induced marrow suppression and it is the cytokine activation that confers the poor prognosis, not the haemoglobin per se. Allopurinol induced intraindividual decreases in haemoglobin within the normal range are most unlikely to be due to allopurinol increasing cytokine activity and hence are unlikely to indicate a poor prognosis. Indeed, they are likely to indicate, if anything, an improved prognosis, as argued above. Furthermore, what we observed here is a haemoglobin shift downwards within the normal range but not into the frankly anaemic range, where the $\mathrm{U}$ shaped curve begins to display harm. In fact in non-CHF populations, a lowered haemoglobin within the normal range is a favourable prognostic sign because it signifies lowered viscosity and hence lowered clotting potential. ${ }^{43}$ It is intriguing that allopurinol reduces the "stickiness" of both the vessel wall (endothelial function) and the blood in the vessel lumen (haemoglobin and viscosity), which conceivably may act together to reduce thrombotic events.

The allopurinol induced fall in total cholesterol was small but previous data do suggest a possible mechanism. Pinelli and colleagues ${ }^{44}$ showed that blocking nitric oxide increased total cholesterol concentrations, implying that treatments that improve endothelial function per se may somehow also have a favourable effect on lipid metabolism.

There are pros and cons to using crossover versus parallel group studies. Narang and colleagues ${ }^{20}$ recommend crossover studies for the initial evaluation of interventions on exercise capacity in heart failure, as here. Fortunately, we saw no order effect in this crossover study for any of the parameters we measured. In accordance with this, baseline values for all parameters were virtually identical at the start of each treatment phase.

In summary, an important negative finding here is that despite high hopes for it, allopurinol does not improve exercise capacity in CHF when given on top of already optimal treatment. However, an important positive finding is that allopurinol has favourable effects on the key prognostic marker BNP. This may be indirect evidence that allopurinol improves LV function, remodelling, or both in human CHF. Therefore, allopurinol improves both key prognostic markers, BNP and endothelial function, which now provides support for the notion that a mortality trial of allopurinol versus placebo may be warranted in CHF, especially since retrospective data also support the possibility that such a trial may be positive if the optimal dose is used. ${ }^{45}$

\section{ACKNOWLEDGEMENTS}

This work was funded by the British Heart Foundation. 


\section{Authors' affiliations}

A D Gavin, A D Struthers, Division of Medicine \& Therapeutics, Ninewells Hospital \& Medical School, Dundee, UK

Neither author has any conflicts of interest

\section{REFERENCES}

1 Cardillo C, Kilcoyne CM, Cannon RO, et al. Xanthine oxidase inhibition with oxypurinol improves endothelial vasodilator function in hypercholesterolaemia but not hypertensive patients. Hypertension 1997:30:57-63.

2 Butler R, Morris AD, Belch JJF, et al. Allopurinol normalises endothelial function in type 2 diabetics with mild hypertension. Hypertension 2000:5:746-51.

3 Guthikonda S, Sinkey C, Barenz T, et al. Xanthine oxidase inhibition reverses endothelial dysfunction in heavy smokers. Circulation 2003;107:416-21.

4 Doehner W, Schoene N, Rauchhaus M, et al. Effects of xanthine oxidase inhibition with allopurinol on endothelial function and peripheral blood flow in hyperuricemic patients with chronic heart failure: results from 2 placebo controlled studies. Circulation 2002;105:2619-24.

5 Farquharson C, Butler R, Hill A, et al. Allopurinol improves endothelial dysfunction in chronic heart failure. Circulation 2002;106:221-6.

6 O'Driscoll G, Green DJ, Rankin JM, et al. Nitric oxide-dependent endothelia function is unaffected by allopurinol in hypercholesterolaemic subjects. Clin Exp Pharmacol Physiol 1999;26:779-83.

7 Drexler H. Endothelium as a therapeutic target in heart failure. Circulation 1998;98:2652-5

8 Hambrecht R, Fiehn E, Weigl C, et al. Regular physical exercise corrects endothelial dysfunction and improves exercise capacity in patients with chronic heart failure. Circulation 1998;98:2709-15.

9 Widlansky ME, Gokce N, Keaney JF, et al. Clinical implications of endothelial dysfunction. J Am Coll Cardiol 2003;42:1149-60.

10 Vita JA, Keaney JF. Endothelial function: a barometer for cardiovascular risk. Circulation 2002; 106:640-2.

11 Schachinger V, Britten MB, Zeiher AM. Prognostic impact of coronary vasodilator dysfunction in adverse long-term outcome of coronary hear disease. Circulation 2000;101:1899-906.

12 Gokce N, Keaney JF, Hunter LM, et al. Risk stratification for postoperative cardiovascular events via non-invasive assessment of endothelial function: a prospective study. Circulation 2002;105:1567-72.

13 Gokce N, Keaney JF, Hunter LM, et al. Predictive value of non-invasively determined endothelial dysfunction for long-term cardiovascular events in patients with peripheral vascular disease. J Am Coll Cardio 2003;410:1769-75.

14 Perticone F, Ceravolo R, Pujia A, et al. Prognostic significance of endothelia dysfunction in hypertensive patients. Circulation 2001;104:191-6.

15 Al Suwaidi J, Hamsaki S, Higano ST, et al. Long term follow up of patients with mild coronary disease and endothelial dysfunction. Circulation 2000;101:948-54.

16 Heitzer T, Schlinzig T, Krohn K, et al. Endothelial dysfunction, oxidative stres and risk of cardiovascular events in patients with coronary artery disease. Circulation 2001; 104:2673-8.

17 Modena MG, Bonetti L, Coppi F, et al. Prognostic role of reversible endothelial dysfunction in hypertensive postmenopausal women. J Am Coll Cardiol 2002;40:505-10

18 Dahlof B, Devereux RB, Kjeldsen SE, et al. Cardiovascular morbidity and mortality in the LIFE: a randomised trial against atenolol. Lancet 2002;359:995-1003

19 Latini R, Masson S, Anand I, et al. The comparative prognostic value of plasma neurohormones at baseline in patients with heart failure enrolled in Val-HeFT. Eur Heart J 2004;25:292-9.

20 Narang R, Swedberg K, Cleland JGF. What is the ideal study design for evaluation of treatment for heart failure? Eur Heart J 1996;17:120-34.

21 Landmesser U, Drexler $\mathrm{H}$. Allopurinol and endothelial function in heart failure future or fantasy? Circulation 2002;106:173-5.

22 Cappola TP, Kass DA, Nelson GS, et al. Allopurinol improves myocardial efficiency in patients with idiopathic dilated cardiomyopathy. Circulation 2001;104:2407-11.

23 McDonagh TA, Cunningham AD, Morrison CE, et al. Leff ventricular dysfunction, natriuretic peptides, and mortality in an urban population. Heart $2001 ; 86: 21-6$
24 Tsutamoto T, Wada A, Maeda K, et al. Plasma brain natriuretic peptide level as a biochemical marker of morbidity and mortality in patients with asymptomatic or minimally symptomatic left ventricular dysfunction. Eur Heart J 1999;20:1799-807.

25 De Lemos JA, Morrow DA, Bentley JH, et al. The prognostic value of B-type natriuretic peptide in patients with acute coronary syndromes. N Engl J Med 2001;345:1014-21

26 Cowie MR, Struthers AD, Wood DA, et al. Value of natriuretic peptides in assessment of patients with possible new heart failure in primary care. Lancet 1997;350:1347-51

27 McGeoch G, Lainchbury J, Town Gl, et al. Plasma brain natriuretic peptide after long-term treatment for heart failure in general practice. Eur $J$ Heart Failure 2002:4:479-83

28 Wang TJ, Larson MG, Levy D, et al. Plasma natriuretic peptide levels and the risk of cardiovascular events and death. N Engl J Med 2004;350:655-63.

29 Nakagami H, Takemoto M, Liao JK. NADPH oxidase derived superoxide anion mediates angiotensin II induced cardiac hypertrophy. J Mol Cell Cardiol 2003;35:851-9.

30 Kinugawa S, Tsutsui $\mathrm{H}$, Hayashidani S, et al. Treatment with dimethylthiourea prevents left ventricular remodeling and failure after experimental myocardial infarction in mice: role of oxidative stress. Circ Res 2000;87:392-8.

31 Mak S, Newton GE. Vitamin C augments the inotropic response to dobutamine in humans with normal left ventricular function. Circulation $2001 ; 103: 826-30$

32 Nakamura R, Egashira K, Machida Y, et al. Probucol attenuates left ventricular dysfunction and remodeling in tachycardia induced heart failure: roles of oxidative stress and inflammation. Circulation 2002;106:362-7.

33 Node K, Fujita M, Kitakaze $M$, et al. Short-term statin therapy improves cardiac function and symptoms in patients with idiopathic dilated cardiomyopathy. Circulation 2003;108:839-43.

34 Takemoto $M$, Node K, Nakagami $H$, et al. Statins as antioxidant therapy for preventing cardiac myocyte hypertrophy. J Clin Invest 2001;108:1429-37.

35 Hayashidani S, Tsutsui H, Shiomi T, et al. Fluvastatin, a 3-hydroxy-3methylglutaryl coenzyme A reductase inhibitor, attenuates left ventricular remodeling and failure after experimental myocardial infarction. Circulation 2002; 105:868-73

36 Patel R, Nagueh SF, Tsybouleva N, et al. Simvastatin induces regression of cardiac hypertrophy and fibrosis and improves cardiac function in a transgenic rabbit model of human hypertrophic cardiomyopathy. Circulation 2001;104:317-24.

37 Wolfrum S, Grimm M, Heidbreder M, et al. Acute reduction of myocardial infarct size by a hydroxymethyl glutaryl coenzyme A reductase inhibitor is mediated by endothelial nitric oxide synthase. J Cardiovasc Pharmacol 2003;41:474-80

38 Di Napoli P, Antonio Taccardi A, Grilli A, et al. Simvastatin reduces reperfusion injury by modulating nitric oxide synthase expression: an ex vivo study in isolated working rat hearts. Cardiovasc Res 2001;51:283-93.

39 Landmesser U, Drexler H. Allopurinol improves LV remodelling after MI. Heart Failure 2003, Strasbourg, 21-24 June, 2003.

40 Giannuzzi P, Temporelli PL, Corra U, for ELVD-CHF study group, et al. Antiremodeling effect of long term exercise training in patients with stable chronic heart failure: results of the exercise in left ventricular dysfunction and chronic heart failure (ELVD-CHF) trial. Circulation 2003;108:554-9.

41 Hartmann F, Packer M, Coats AJS, et al. Prognostic impact of plasma N terminal pro brain natriuretic peptide in severe chronic congestive heart failure. A substudy of the COPERNICUS trial. Circulation 2004;1 10:1780-6.

42 de Bold AJ, Borenstein HB, Veress AT, et al. A rapid and potent natriuretic response to intravenous injection of atrial myocardial extract in rats. Life Sciences 1981;28:89-94.

43 Danesh J, Collins R, Peto R, et al. Haematocrit, viscosity, erythrocyte sedimentation rate: meta-analyses of prospective studies of coronary heart disease. Eur Heart J 2000;21:515-20.

44 Pinelli A, Trivulzio S, Tomasoni L, et al. Drugs modifying nitric oxide metabolism affect plasma cholesterol levels, coagulation parameters, blood pressure values and the appearance of plasma myocardial necrosis markers in rabbits: opposite effects of L-NAME and nitroglycerine. Cardiovasc Drugs Ther 2003;17:15-23.

45 Struthers AD, Donnan PT, Lindsay P, et al. The effect of allopurinol on mortality and hospitalisation in chronic heart failure: a retrospective cohort study. Heart 2002;87:229-34 